\title{
In-situ 3D CT-XAFS Imaging of Pt/C Cathode Catalysts in Polymer Electrolyte Fuel Cell during Degradation Processes by Anode Gas Exchange Cycles
}

$\underline{\text { Kotaro Higashi }}^{1}$, Tomohiro Sakata ${ }^{1}$, Oki Sekizawa ${ }^{1,2}$, Nozomu Ishiguro ${ }^{3}$, Gabor Samjeskè ${ }^{1}$, Shinobu Takao $^{1}$, Takuma Kaneko ${ }^{1}$, Tomoya Uruga ${ }^{1,2}$, Yasuhiro Iwasawa ${ }^{1, *}$ and Mizuki Tada ${ }^{3,4}$

1. Innovation Research Center for Fuel cells, The University of Electro-Communications, Chofu, Tokyo 182-8585, Japan

2. Japan Synchrotron Radiation Research Institute, SPring-8, Sayo, Hyogo 679-5198, Japan

3. RIKEN Harima Branch, 1-1-1 Kouto, Sayo, Hyogo 679-5148 Japan

4. Nagoya University, Furo-cho, Chikusa-ku, Nagoya, Aichi 464-8602, Japan

* Corresponding author, iwasawa@pc.uec.ac.jp

Polymer electrolyte fuel cell (PEFC) is one of the most efficient clean energy generation systems with high power density at low temperatures, which has a great advantage for its automotive applications. However, a major problem is practically insufficient durability of the membrane electrode assembly (MEA) in PEFC under the operating conditions, especially during startup/shutdown cycles of PEFC, where the feed gases are exchanged. When the anode feed gas exchange cycles are repeated, while keeping the cathode under air, the cathode catalyst degradation proceeds [1,2]. To visualize 3D degradation of the cathode catalyst layer, we measured the structural and electronic changes of Pt nanoparticles in the PEFC MEA during the anode-gas exchange (AGEX) cycles by in-situ CT-XAFS imaging technique $[3,4]$ at our beamline BL36XU in SPring-8.

A custom-made fuel-cell was equipped with a $9.9 \mathrm{~cm}^{2}$ MEA composed of $0.6 \mathrm{mg}-\mathrm{Pt} / \mathrm{cm}^{2}$ (TEC10E50E, Tanaka Kikinzoku Kogyo) as a cathode catalyst and $0.6 \mathrm{mg}-\mathrm{Ru} / \mathrm{cm}^{2}$ (TECRu(ONLY)E50, Tanaka Kikinzoku Kogyo) as an anode catalyst with a Nafion (NR-212, Sigma-Aldrich) electrolyte membrane. After the steady-state operation - $\left(\mathrm{H}_{2} /\right.$ air $)$ at the MEA for $30 \mathrm{~s}$, anode $\mathrm{H}_{2}$ gas was switched to air, and this shutdown condition (air/air) was kept for $60 \mathrm{~s}$. Electrochemical experiments and in-situ CT-XAFS imaging were conducted after the aging treatment and after every 100 AGEX cycles. In-situ CT-XAFS measurements were performed under the cell potentials of $0.4 \mathrm{~V}$ and $1.0 \mathrm{~V}$ vs RHE in $\mathrm{H}_{2}$ (anode) and $\mathrm{N}_{2}$ (cathode), $\sim 93 \%$ relative humidity atmospheres. The CT imaging was performed in the space of $667 \mu \mathrm{m}$ cubes and at a spatial resolution of $2.5 \mu \mathrm{m}$. The rotation angle of the PEFC was restricted from -80 to $+80^{\circ}$. To reduce X-ray damage on MEA during the CT-XAFS measurements, we adjusted X-ray flux by $\mathrm{Al}$ absorber.

Figure 1 shows the 3D images of the cathode catalyst layer at the cell potential of $1.0 \mathrm{~V}$ after aging treatment. It is seen that the distribution of $\mathrm{Pt}$ amount was inhomogeneous in the initial state. Figure 2 shows the 3D images of Pt amount and Pt valence at the same position of Figure 1 after AGEX 300 cycles at the cell potential of $1.0 \mathrm{~V}$. The Pt valence at $1.0 \mathrm{~V}$ decreased after AGEX 300 cycles. Very recently, we reported the fractions of less and inactive Pt nanoparticles for the electrochemical reaction increased by increasing AGEX cycles by in-situ time-resolved XAFS [2]. Figure 2 also indicates a decrease in the fraction of the active Pt catalyst nanoparticles by the AGEX cycles. The results by the AGEX treatment are also consistent with those observed in our previous ex-situ TEM studies [1], where we found that the Pt catalyst nanoparticles did not change significantly and the carbon support was corroded locally but carbon cracks did not extend. On the other hands, the Pt amount and crack regions in the cathode catalyst layer almost unchanged, which is quite different from that observed by our previous CT-XAFS 
measurements after $0.6-1.0 \mathrm{~V}$ rectangular accelerated durability test (ADT) treatment [4]. The current 3D CT-XAFS imaging provides a new piece of information on 3D degradation patterns of Pt nanoparticles that reflect origin and history of MEA Pt/C degradations.

\section{References:}

[1] G. Samjeské, K. Higashi, S. Takao, Y. Iwasawa, et al, ChemElectroChem 2 (2015) 1595.

[2] K. Higashi, G. Samjeské, S. Takao, Y. Iwasawa, et al, J. Phys. Chem. C 121 (2017) 22164.

[3] O. Sekizawa, T. Uruga, N. Ishiguro, M. Tada, et al, J. Phys.: Conf. Ser. 849 (2017) 012022.

[4] H. Matsui, N. Ishiguro, T. Uruga, M. Tada, et al, Angew. Chem. Int. Ed. 56, (2017) 9371.

[5] This study was performed with the approval of SPring-8 proposals (2015A7805, 2015B7805, 2016A7805, 2016B7805, 2017A7805, 2017A7840, 2017B7840). This work was supported by JSPS KAKENHI Grant Number JP15K17807 and the New Energy and Industrial Technology Development Organization (NEDO) of Japan.

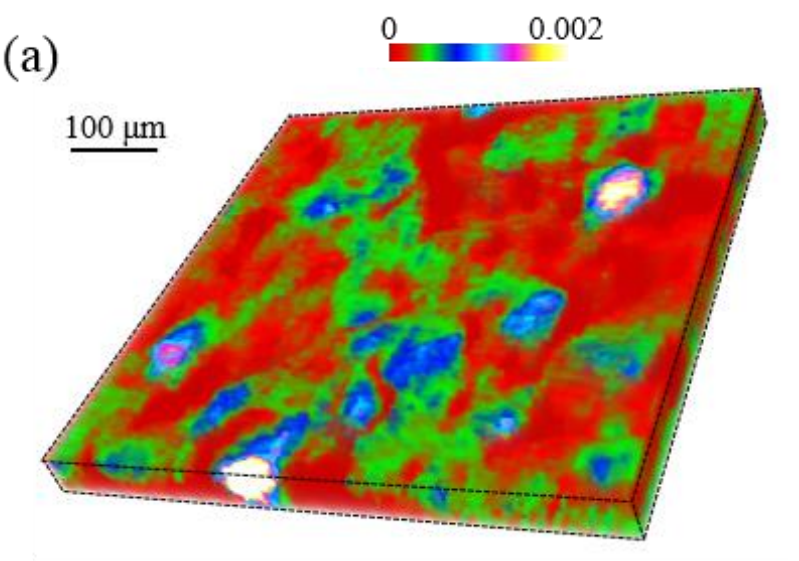

(b)

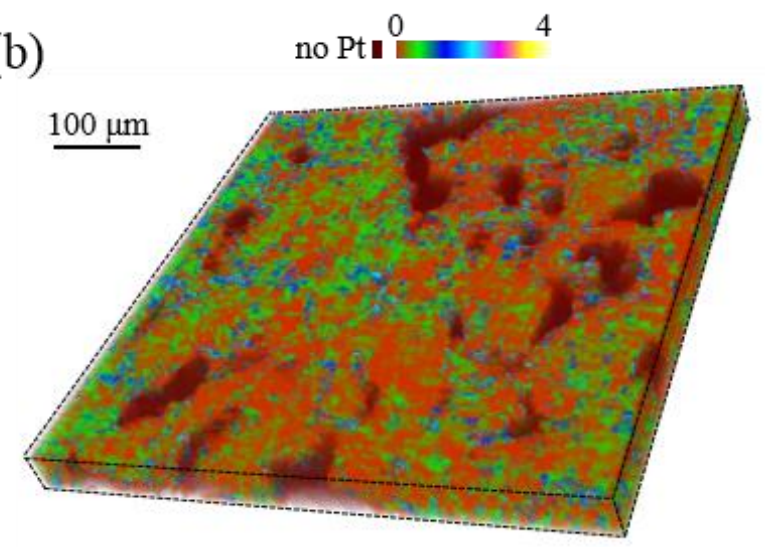

Figure. 1. 3D images of cathode catalyst layer at the cell potential $1.0 \mathrm{~V}$ after aging treatment. (a) Pt amount and (b) Pt valence.
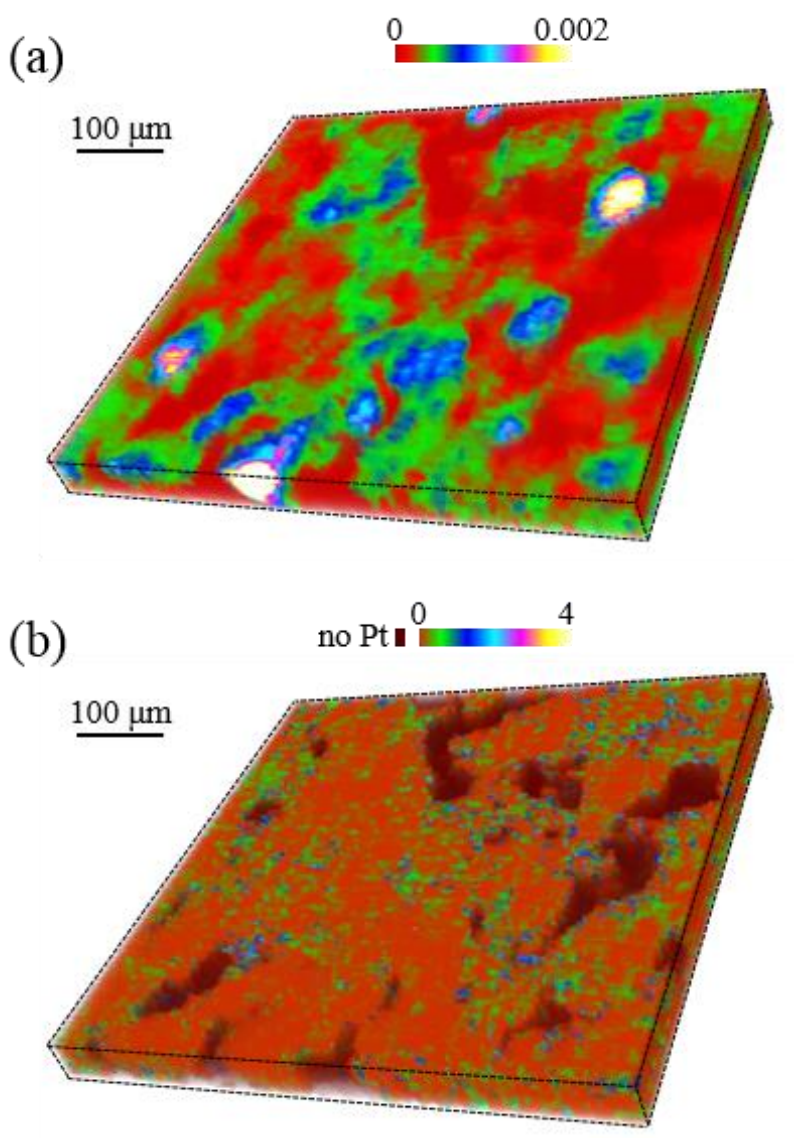

Figure. 2. 3D images of cathode catalyst layer at the cell potential $1.0 \mathrm{~V}$ after AGEX 300 cycles. (a) Pt amount and (b) Pt valence. 